\title{
Association between Elevated Serum Ferritin and HBA1C in Type 2 Diabetes Mellitus
}

\author{
Priyanka Vaitla ${ }^{1}$, MD Sabiullah ${ }^{2}$, N.Vani ${ }^{3}$ \\ ${ }^{I}$ (Department of Biochemistry, Osmania Medical College, India) \\ ${ }^{2}$ (Department of Biochemistry, Osmania Medical College, India) \\ ${ }^{3}$ (Department of Biochemistry, Osmania Medical College, India)
}

\begin{abstract}
:
Background and objectives : Diabetes is a disease of metabolic dysregulation. Recent studies have emphasized the role of serum ferritin in insulin resistance and the incidence of diabetes mellitus. However, the role of ferritin as a marker of iron overload in pancreatic damage and peripheral insulin resistance or its role as an inflammatory marker is not clear. The aim of this study is to establish an association between serum ferritin, FBS and HbAlc in type 2 diabetes mellitus and to evaluate the role of serum ferritin on glycemic status.

Methods : A case control study was done with 90 type 2 DM patients. FBS,PLBS, Hb\%, HbAlc\% and serum ferritin were measured.

Results : Significant hyperglycemia was observed in the cases compared to the controls. Mean $\pm S D$ of FBS, PLBS, HbAlc and serum ferritin were highest in group with poor glycemic control.

Interpretations and conclusion: Higher positive correlation of serum ferritin with HbAlc shows that dysglycemia affects ferritin levels possibly due to inflammation or oxidative stress or a combination of two. Iron may negatively impact on glycemic control. Also in the presence of hyperglycemia and inflammation, iron may contribute to the development and progression of oxidative injury.
\end{abstract}

Keywords: Diabetes mellitus, ferritin, oxidative stress, HbAlc

\section{Introduction}

Diabetes is a complex metabolic disease characterized by a state of chronic hyperglycemia. This state of hyperglycemia is considered to be resulted from a diversity of etiologies, environmental and genetic; acting jointly. India leads the world with largest number of diabetic subjects earning the dubious distinction of being termed the "diabetes capital of the world". Insulin resistance plays a major role in the development of IGT and diabetes. Insulin resistance is manifested by decreased insulin mediated glucose uptake and metabolism in adipocytes and skeletal muscle and by impaired suppression of hepatic glucose output.

The symptoms and longterm complications are influenced by the average blood glucose levels. Amongst the various markers of glycemic control, glycated hemoglobin has now been established as the most reliable, reflecting average glucose concentration over the previous two to three months. Emerging scientific evidences has disclosed unsuspected influence between iron metabolism and type 2 diabetes. The ability of iron to reversibly oxidize and reduce, makes it potentially hazardous because of its ability to generate powerful oxidant species such as hydroxyl radicals.The relationship is bi-directional - iron affects glucose metabolism, and glucose metabolism impinges on several iron metabolic pathways. Insulin influences the iron uptake and storage by increasing the cell surface transferrin receptors, reciprocally iron influences the insulin activity by interfering with glucose uptake and utilization. Iron causes hyperinsulinemia by decreasing the insulin uptake and metabolism by hepatocytes. Iron in its free form i.e., in non-transferrin bound form is known to induce oxidation of biomolecules through Heber-Weiss and Fenton reactions by producing harmful hydroxyl radicals $[1]$.

Iron homeostasis in the body can be evaluated by measuring Ferritin, a key protein which regulates the homeostasis. ${ }^{[2]}$ Increased serum ferritin, reflecting body iron overload, is often associated with measures of insulin resistance, such as elevated blood glucose and insulin levels. Elevated iron stores may induce diabetes through a variety of mechanisms, including oxidative damage to pancreatic beta cells, impairment of hepatic insulin extraction by the liver, and interference with insulin's ability to suppress hepatic glucose production. Hence, the present study was carried out to establish relationship between serum ferritin and type 2 diabetes mellitus.

\section{Materials And Methods}

A case control study was conducted in the Department of Biochemistry, Osmania General Hospital, Hyderabad. The study was conducted over a period of one year. 90 type 2 diabetes patients and controls were included in the study. The cases and samples were collected from Department of Endocrinology, Osmania 
General Hospital. Investigations were performed at the Department of Biochemistry, Osmania Medical College/Osmania General Hospital, Hyderabad.

\title{
2.1 Inclusion criteria
}

Group 1 included healthy controls who were matched for age and sex.

Group 2 included those individuals with diagnosed Type 2 Diabetes mellitus patients with $\mathrm{HbA} 1 \mathrm{c}<.8 \%$ and without complications

Group 3 included those individuals with diagnosed Type 2 Diabetes mellitus patients with HbA1c $>8 \%$ or with complications

\author{
2.2 Exclusion criteria \\ Individuals with \\ - Anemia \\ - Hypertension \\ - Overt thyroid dysfunction \\ - Chronic kidney disease \\ - Chronic liver disease \\ $\bigcirc$ On corticosteroid therapy
}

\subsection{Data collection}

Proforma including age, sex, medical history, onset, duration and complications of Diabetes was filled. Physical examination was done measuring height, weight, waist circumference and Blood pressure was recorded. Fasting venous blood sample and postprandial venous blood sample were collected from all the subjects. Laboratory parameters including FBS, PLBS, serum ferritin, Hemoglobin, Renal function tests and liver function tests were estimated in the samples.

\section{Statistical Analysis}

The data was analyzed using GraphPad Prism software version 6.0. Descriptive results are expressed as mean and SD of various parameters in different groups. to assess the significance of the differences observed in the mean values of different parameters observed in different groups studied, the data is subjected to ANOVA test. The significance of difference of mean values of different groups and within the groups is represented by $\mathrm{p}$ values and $\mathrm{p}$ value $<0.05$ is considered as significant. Pearson's correlation was done to assess the correlation of parameters within each group.

\section{Results}

Serum ferritin was significantly higher in cases compared to controls $(\mathrm{p}<0.001)$. In group 2 , ferritin was positively correlated with FBS and PLBS but was not statistically significant. In group 3, ferritin was positively correlated with FBS and PLBS but only correlation with PLBS was statistically significant. Ferritin was positively correlated with $\mathrm{HbAlc}$ and was statistically significant in group 2 and group 3 . Results show that serum ferritin increased significantly in diabetes mellitus patients with high HbAlc or with complications compared to those without complications. There was no correlation observed between serum ferritin and other parameters

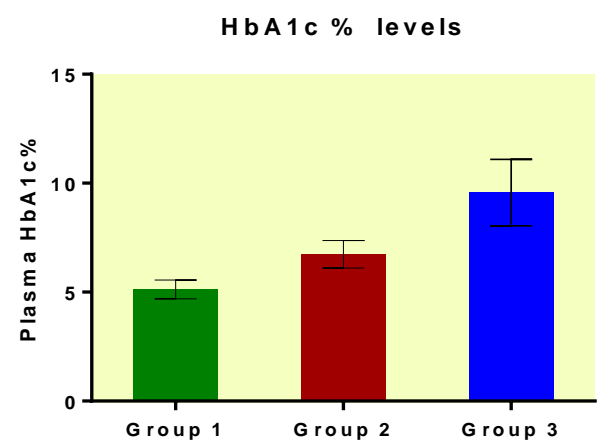

Fig1:graphical representation of mean \pm sd of hba1c \% in the 3 groups 


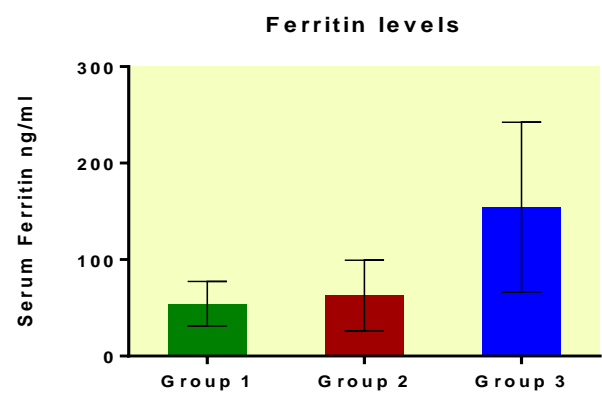

Fig2: graphical representation of mean \pm sd of serum ferritin in 3 groups

\section{Discussion}

Oxidative stress has been implicated in the pathogenesis of the complications seen in type $2 \mathrm{DM}^{[3]}$. Iron in moderate quantities is essential for all cell metabolism and growth, but it is toxic when unleashed by protein $^{[4]}$. Although the exact mechanism of iron induced diabetes is uncertain, it is likely, to be mediated by three key mechanisms: Insulin deficiency, insulin resistance and hepatic dysfunction ${ }^{[5]}$. In conditions of iron overload there is generally an increase in the expression of intracellular L-subunit rich ferritins, paralleled by an increase in these ferritins in the plasma ${ }^{[6]}$. Serum ferritin was significantly higher in group 3 compared to group 1 and 2. Different theories regarding the role of ferritin in DM have been suggested. Ferritin has been referred as a marker for insulin resistance possibly due to iron deposition in the liver leading to hepatic insulin resistance and increased hepatic glucose production ${ }^{[7],[8]}$. Others have determined ferritin just as a marker of pancreatic inflammation, while pancreatic damage due to some degree of subclinical hemochromatosis has been considered in some cases of diabetes ${ }^{[9]}$. Two large epidemiological studies reported a strong association between elevated serum ferritin concentration and increased risk for diabetes ${ }^{[10],[11]}$. Elevated iron stores may be associated with impaired glycemic control through a variety of mechanisms, including oxidative damage to pancreatic beta cells, impairment of hepatic insulin extraction by the liver, and interference with insulin's ability to suppress hepatic glucose production.Raised Serum Ferritin may possibly be related to the occurrence of long term complications of diabetes, both micro vascular and macro vascular ${ }^{81}$. Sumesh Raj et al in their study found that serum ferritin, a reflector of body iron stores was significantly higher in diabetic patients when compared to controls and this significantly increased as duration of diabetes increased ${ }^{[12]}$. This possibly reflects the subclinical hemochromatosis developing in a long standing diabetic patient. ${ }^{[13]}$ In their study, there was a positive correlation between serum ferritin and FBS, HbA1c.This reflected the relation between serum ferritin and glycemic control, both short term and long term.

\section{Conclusion}

It is clear from the results of the present study that increased serum ferritin levels are associated with increased HbA1c reflecting poor glycemic control. We suggest that serum ferritin can be used as an inflammatory marker for longterm complications in Diabetes mellitus. Iron may also negatively impact on glycemic control. Though we recommend further studies in this regard, we suggest that ideally the clinical approach should be a careful balance between insufficient iron stores and excess iron.

\section{References}

[1] Nitin S. HbA1c and factors other than diabetes mellitus affecting it. Singapore Med Journal 2010; 51: 616-622.

[2] Jiang R, Manson JE, Meigs JB, Ma J, Rifai N, et al. (2004) Body iron stores in relation to risk of type 2 diabetes in apparently healthy women. JAMA 291: 711-717.

[3] Radoi V, Lixandru D, Mohara M, Virgolici B. Advanced glycation and products in diabetes melltus. Mechanism of action and focused treatment. Proc.Rom.Acad. Series B,2012; 1:9-19.

[4] Herbert V. Everyone should be tested for iron disorders. J Am Diet Assoc 1992;92:1502-1509.76

[5] Swaminathan S, Fonseca VA, Alam MG, Shah SV. The role of iron in diabetes and its complications. Diabetes care. 2007;30(70:1926-1933)

[6] Halliday JW, Mack U, Powell LW. The kinetics of serum and tissue ferritins: relation to carbohydrate content. Br J Haematol. 1979; 42(40): 535-546.

[7] Forouhi NG, Harding AH, Allison M, Sandhu MS, Welch A, Luben R; et al Elevated serum ferritin levels predict new- onset type 2 diabetes: results from the EPIC-Norfolk prospective study. Diabetologia. 2007;50:949-956.

[8] Fernandez-Real JM, Ricart-Engel W, Arroyo E, Balanca R, Casamitjana-Abella R, et al. Serum ferritin as a component of the insulin resistance syndrome. Diabetes Care. 1998;21(1):62-68.

[9] Sharifi F and Sazandeh Sh. Serum ferritin in type 2 diabetes mellitus and its relationship with HbA1c. Acta Med Iranica. 2004; 42(2):142-145.

[10] Ford ES, Cogswell ME. Diabetes and serum ferritin concentration among U.S adults. Diabetes Care. 1999;22:1978-83.

[11] Tuomainen TP, Nyyssonen K, Salonen R, Tervahauta A, Korpela H, Lakka T, et al. Body iron stores are associated with serum insulin and blood glucose concentrations. Population study in 1013 eastern Finnish men. Diabetes Care. 1997;22:1978-83.

[12] Sumesh Raj, G. V. Rajan: Correlation between elevated serum ferritin and HbA1c in type 2 diabetes mellitus. International Journal of Research in Medical Sciences Raj S et al. Int J Res Med Sci. 2013 Feb;1(1):12-15

[13] Moczulski DK, Grzeszczak W, Gawlik B. Role of hemochromatosis C282Y and H63D mutations in HFE gene in development of type 2 diabetes and diabetic nephropathy. Diabetes Care 2001;24:1187-91. 